\title{
Research on Removal of Manganese in Drinking Water by Ferric Oxyhydroxides Composites
}

\author{
Haishan Dong1, a, Long Yang',b, Xianggui Zeng ${ }^{3, c}$, Peng Yan ${ }^{4, d}$, Zhicong Yin ${ }^{5, e}$ \\ 1,2,4,5 Faculty of Geosciences and Environmental Engineering, Southwest Jiaotong University, \\ Chengdu Sichuan \\ ${ }^{3}$ Deyang environmental monitoring center station, Deyang Sichuan \\ a1305080092@qq.com, b1320496379@qq.com, c1055713599@qq.com, d359297914@qq.com, \\ e812267987@qq.com
}

\begin{abstract}
Keywords: ferric oxyhydroxide; drinking water; zeolite; potassium permanganate; sodium hypochlorite.

Abstract: By immobilized ferric oxyhydroxide on absorbent can improve its dispersion property in aqueous solution, then improve its application in water treatment. Ferric oxyhydroxides composites had been used as the absorbent on contaminants removing nowadays. This paper use this composites to absorb manganese to improve its local concentration, then use $\mathrm{KMnO} 4$ and $\mathrm{NaClO}$ as oxidizers to remove manganese in drinking water. The results show that immobilizing ferric oxyhydroxide can improve the carrier absorbent effect, the removal efficiency of manganese reaches $98 \%$ with properly oxidizers added amounts.
\end{abstract}

\section{Introduction}

Nowadays, the process of manganese removal from drinking water mostly adopts contact oxidation process, that is, manganese sand is used as filter material, and manganese is removed by oxidation process $^{[1]}$. In the presence of adsorbent, adsorbent first adsorption of pollutants, improve the local concentration of pollutants. At this point, the addition of oxidants can remove pollutants more efficiently. But the mature time of manganese sand is difficult to control, and the effect is not good when dealing with high concentration raw water ${ }^{[2][3]}$. Hydroxy iron oxide is a kind of iron oxide with large specific surface area, fine particle structure and stable properties. It has been paid more and more attention in water treatment ${ }^{[4]}$. Some scholars use zeolite ${ }^{[5]}$, diatomite ${ }^{[6]}$, quartz sand, bentonite, ${ }^{[7]}$ and other adsorbents to support hydroxy iron oxide to enhance its dispersion in aqueous solution, so as to improve its application in water treatment. Some scholars have made use of the supported hydroxy iron oxide complex to adsorb organic compounds or heavy metals in water, and some achievements have been achieved.

This paper discusses the test of zeolite / ferric hydroxide complexes as catalyst, Potassium Permanganate or sodium hypochlorite as oxidant, the removal of manganese in drinking water by catalytic oxidation. The optimum dosage of oxidant was determined by studying the removal effect.

\section{Text}

Water samples containing manganese. In this experiment, deionized water was used as raw water. By adding a quantitative amount of manganese sulfate and ferrous sulfate reserves, so that the concentration of iron and manganese in raw water $2 \mathrm{mg} / \mathrm{L}$.

Preparation of catalysts. Experiments were carried out by adding $20.0 \mathrm{~g}$ of the washed powdered zeolite to the appropriate amount of $\mathrm{Fe}\left(\mathrm{NO}_{3}\right)_{3}$ solution and then slowly stirring the mixture on the stirrer to allow the zeolite to be thoroughly mixed with the iron-containing mixture. At the same time, the appropriate amount of potassium hydroxide solution was added dropwise at a rate of $10 \mathrm{~mL} / \mathrm{min}$ to ensure that the molar ratio of potassium hydroxide to ferric nitrate was $2 \mathrm{OH}^{-}: \mathrm{Fe}^{3+}=2.5: 1$. After the drip is finished, place the beaker in a constant temperature water bath at 60 degrees. After setting aside the $12 \mathrm{~h}$, the water in the beaker is evaporated. Rinse the beaker with deionized water and stir it 
with a glass bar to mix the solid suspension with deionized water. Finally, sit and observe. At this point, it is found that the settling of the suspended object is fast and the stratification is obvious. The supernatant was poured into the deionized water and the cleaning process was repeated several times until the supernatant $\mathrm{pH}$ value was $<8$. To filter the mixture into the cake, 105 degrees oven drying ground for $4 \mathrm{~h}$.

Experimental method. This experiment uses a static beaker test. In the experiment, $400 \mathrm{~mL}$ of iron-containing raw water was put into $500 \mathrm{~mL}$ beaker, then the catalyst was added, placed on a hexagonal blender, and the appropriate amount of oxidizing agent was stirred at a speed of 300r / min and started to stand. In the course of the experiment, the supernatant was taken periodically to determine the optimum reaction time and the optimum amount of oxidant dosing.

The concentration of manganese ions in this test was determined by flame atomic absorption spectrophotometer.

\section{Results and discussion of the experiment}

Effect of zeolite as catalyst on manganese removal by oxidation. In order to compare the load after hydroxyl iron oxide carrier on manganese removal ability of ascension, the experiment firstly using zeolite as catalyst, catalyst dosing quantity $0.5 \mathrm{~g}$, potassium permanganate and sodium hypochlorite oxidant, and adjust the raw water $\mathrm{pH}$ value of 7.5 , the reaction time of $3 \mathrm{~h}$. The zeolite was used as the unmodified zeolite.

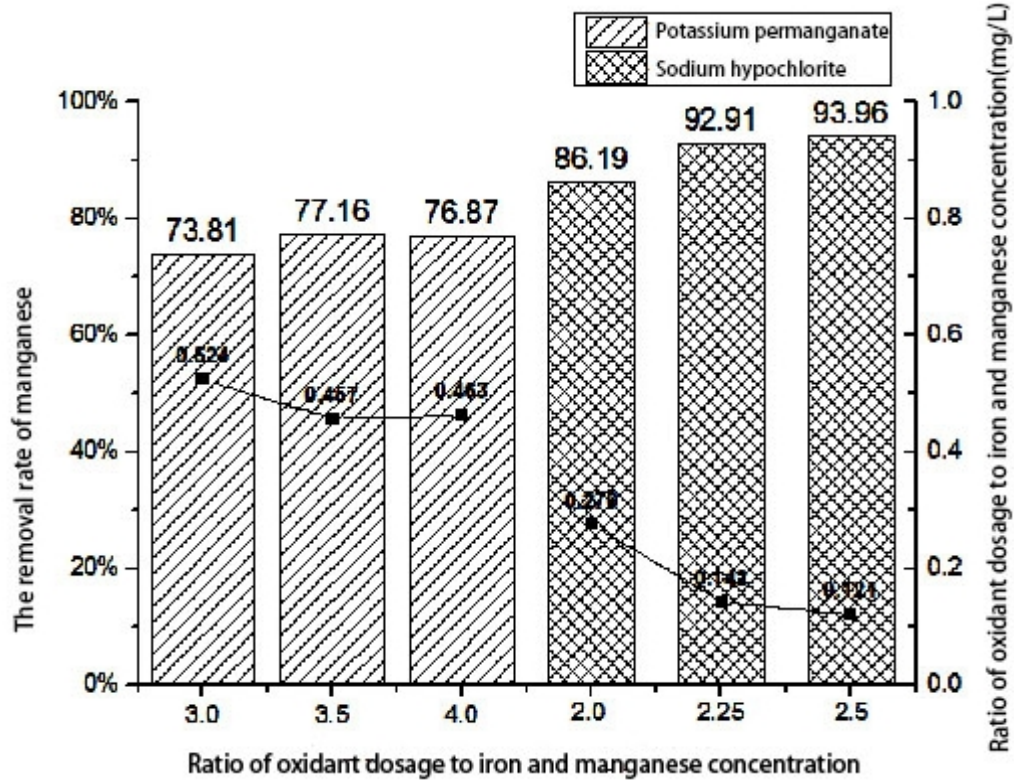

Fig.1 The effect of zeolite as catalyst on manganese removal by oxidation

As can be seen from Fig. 1, the efficiency of manganese removal by zeolite as oxidant is generally not up to standard. Among them, the removal effect is poor by Potassium Permanganate as the oxidant, three water samples of water is still pale pink, that Potassium Permanganate and manganese oxidizing agent in the water did not react completely, the removal efficiency is lower than $78 \%$, the standard gap distance. Sodium hypochlorite as oxidant is Potassium Permanganate slightly better, the highest removal rate reached $93.96 \%$, but the water has not yet reached.

Effect of zeolite supported hydroxyl iron oxide composite on manganese removal by oxidation. To verify with zeolite/hydroxyl iron oxide as catalyst, the oxidation of potassium permanganate and sodium hypochlorite as oxidant manganese removal effect, and determine the optimal dosing quantity of oxidant, dosing range, test in catalyst dosing quantity and agitator rotational speed is constant, change the dosing quantity oxidant, measure oxidant by the amount of residual bivalent manganese optimum dosing quantity. At the same time, the speed and time of reaction can be determined by timing sampling. 
Potassium permanganate as an oxidant. The test was first used as an oxidant in potassium permanganate to explore the effect of the catalyst on the removal of manganese and to determine the suitable amount of oxidizing agent. In the experiment, the amount of catalyst was $0.5 \mathrm{~g}$, and the potassium permanganate was from 0 to 3.5 , and the $\mathrm{pH}$ of the original water was 7.5 , and the reaction time was $30 \mathrm{~min}$.

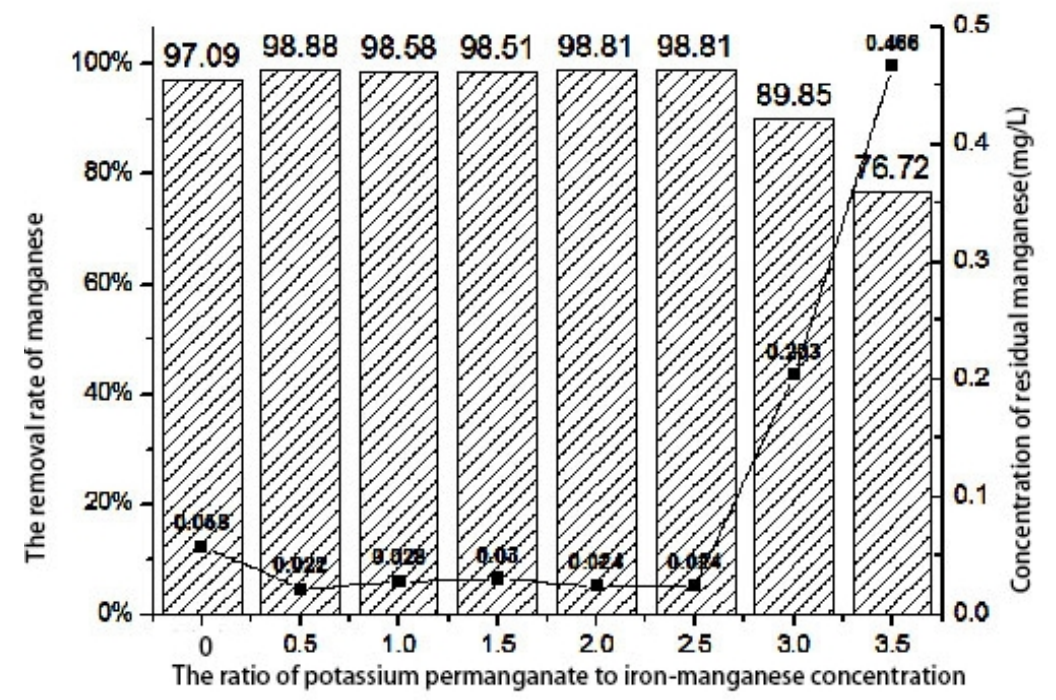

Fig.2 Potassium permanganate as the final manganese removal effect of oxidants

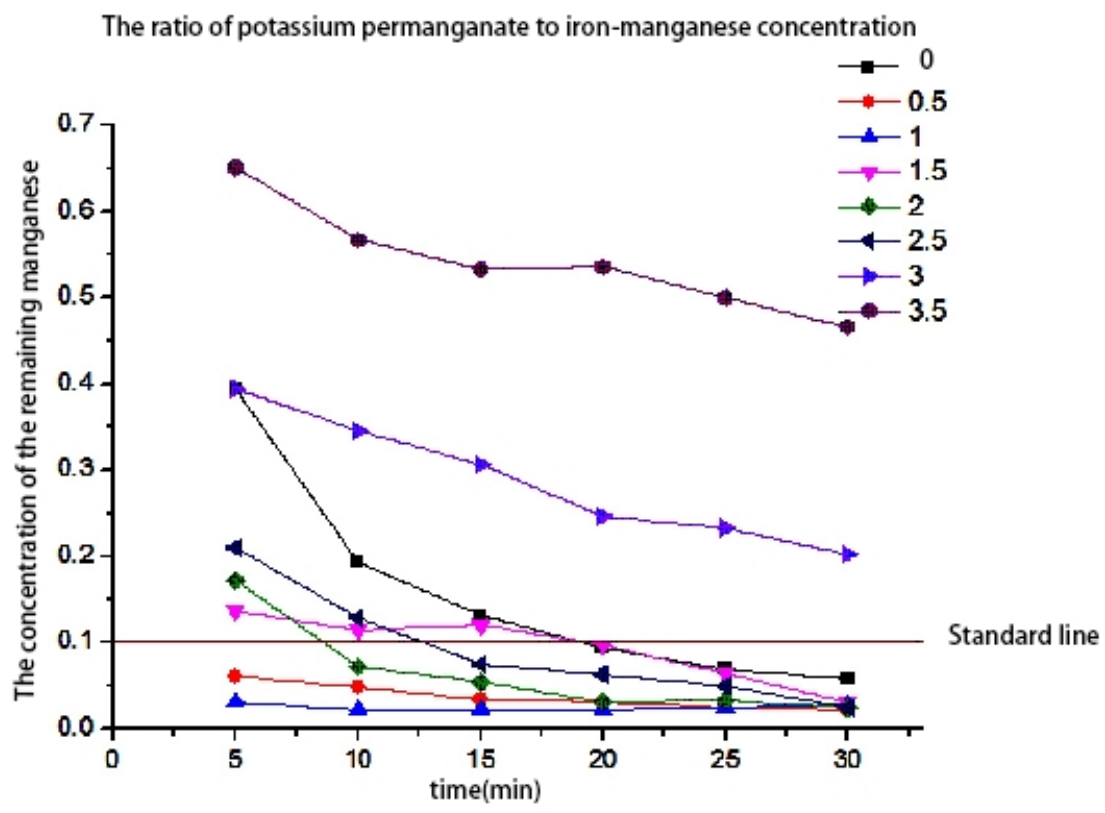

Fig.3 Potassium permanganate as oxidant when the concentration of manganese with time curve

As you can see from the Fig.2 and Fig.3, When not adding Potassium Permanganate, Only by the adsorption of zeolite / hydroxyl iron oxide can the manganese concentration be achieved, The concentration of the remaining manganese in water decreased with time, At 30min, the effluent manganese concentration is $0.058 \mathrm{mg} / \mathrm{L}$, and the removal rate is $97.09 \%$, This shows that zeolite / hydroxyl iron oxide complex has a strong adsorption effect on manganese ions in water, And because the zeolite / hydroxyl iron oxide composite has a strong settling property, when the reaction is completed, the supernatant is clarified and transparent. Zeolite / hydroxyl iron oxide itself has strong adsorption, The subsequent test added the Potassium Permanganate investment and oxidant dosing ratio from 0.5 to 3.5, To do the advanced treatment of manganese, and strive to use the concentration of water and manganese to reach the trace, so as to achieve the requirements of advanced treatment. We can see from Figure 2, when the dosing ratio at 0.5 2.5, the effluent concentration of manganese 
reached trace, removal rate of nearly $99 \%$, When the dosing ratio was $0.5,1$, Mn concentration in the $5 \mathrm{~min}$ are standard, most $\mathrm{Mn}$ in $5 \mathrm{~min}$ have been removed, With the development of time, the concentration of manganese is still decreasing, reaching the lowest point around 15 20min, and then there is a small desorption phenomenon. When the dosing ratio in 1.5 2.5, because Potassium Permanganate investment increased dosage, concentration of manganese in water increased, Continued to decline in $5 \mathrm{~min} \sim 30 \mathrm{~min}$, at the end of the 30min reaction, the effluent manganese concentration can reach the standard, In fact, when shots Gaby in this range, Potassium Permanganate has added excess, the adsorption effect of zeolite / FeOOH strength, the effluent concentration of manganese can still reach. When the dosing ratio is greater than $330 \mathrm{~min}$, the effluent concentration of manganese is not, this is because Potassium Permanganate excessive dosage, the concentration of residual manganese exceeded the adsorption capacity of zeolite / iron oxyhydroxide. We can see from Figure 3, the zeolite / FeOOH can adsorb effective manganese, and fast, to reach the standard in 30min, in the presence of small amounts of oxidant in Potassium Permanganate's case, the effluent can reach the stability of Trace Manganese, Potassium Permanganate's best dosing dosing ratio is $0.5 \sim 1$.

Sodium hypochlorite acts as an oxidizing agent. The experiment shows that potassium permanganate can effectively oxidize manganese in water. At the same time, another strong oxidant, sodium hypochlorite, was used to oxidize two valent manganese in water. At this time, the concentration of iron manganese in the original water was still $2 \mathrm{mg} / \mathrm{L}$, and the catalyst was $0.5 \mathrm{~g}$, while the sodium hypochlorite was 0 to 2.0 , the $\mathrm{pH}$ of the original water was 7.5 and the reaction time was 30min.

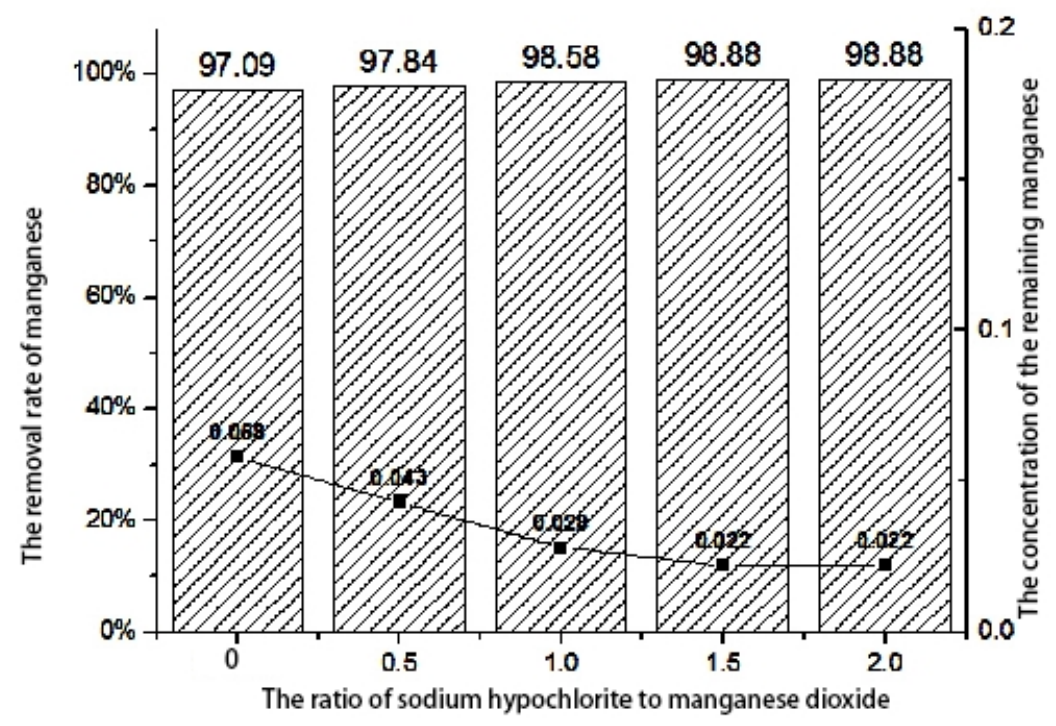

Fig.4 The final removal of sodium hypochlorite as an oxidant

Fig. 4 shows the effect of different sodium hypochlorite on the results of manganese. 


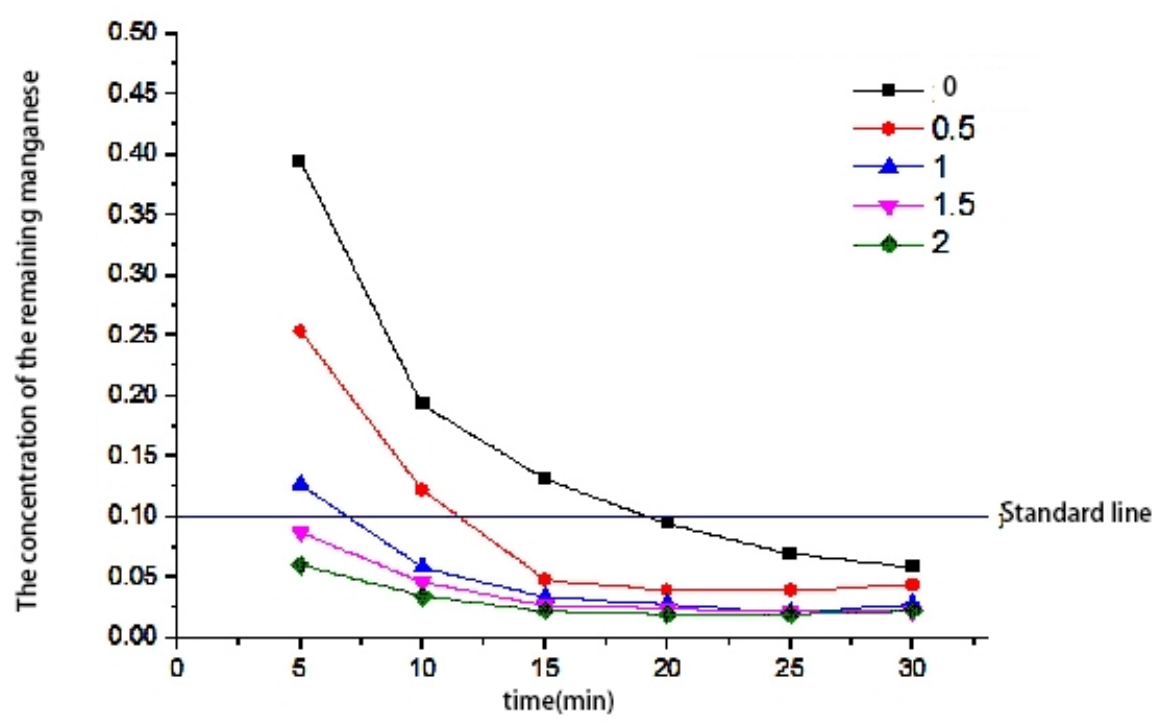

Fig. 5 The variation of manganese concentration in the time of oxidizing sodium hypochlorite as an oxidant

Fig.5 shows that manganese removal rate increased with the increase of sodium hypochlorite dosing quantity increases, within $25 \mathrm{~min}$, the concentration of manganese as time continues to decline, at about 25 min to complete reaction, after 25 minutes, with a little rebound, manganese concentration effluent concentration of manganese can reach the standard. In this case, the concentration of effluent manganese can reach trace amount when the ratio is greater than 1 . The test showed that sodium hypochlorite could be used as a stronger oxidizer to oxidize the manganese ions in the water and remove it. Its oxidation speed is fast, within 30min can be completed, and the removal efficiency is good, the removal efficiency can reach $98.8 \%$.

Comparison of the effect of different technical routes on the effect of manganese. In this paper, the results of the different technical routes were compared that the optimal conditions for the removal of manganese were selected. In order to be consistent with the previous experiment, zeolite adsorption was used in six mixers, rather than thermostatic oscillators.

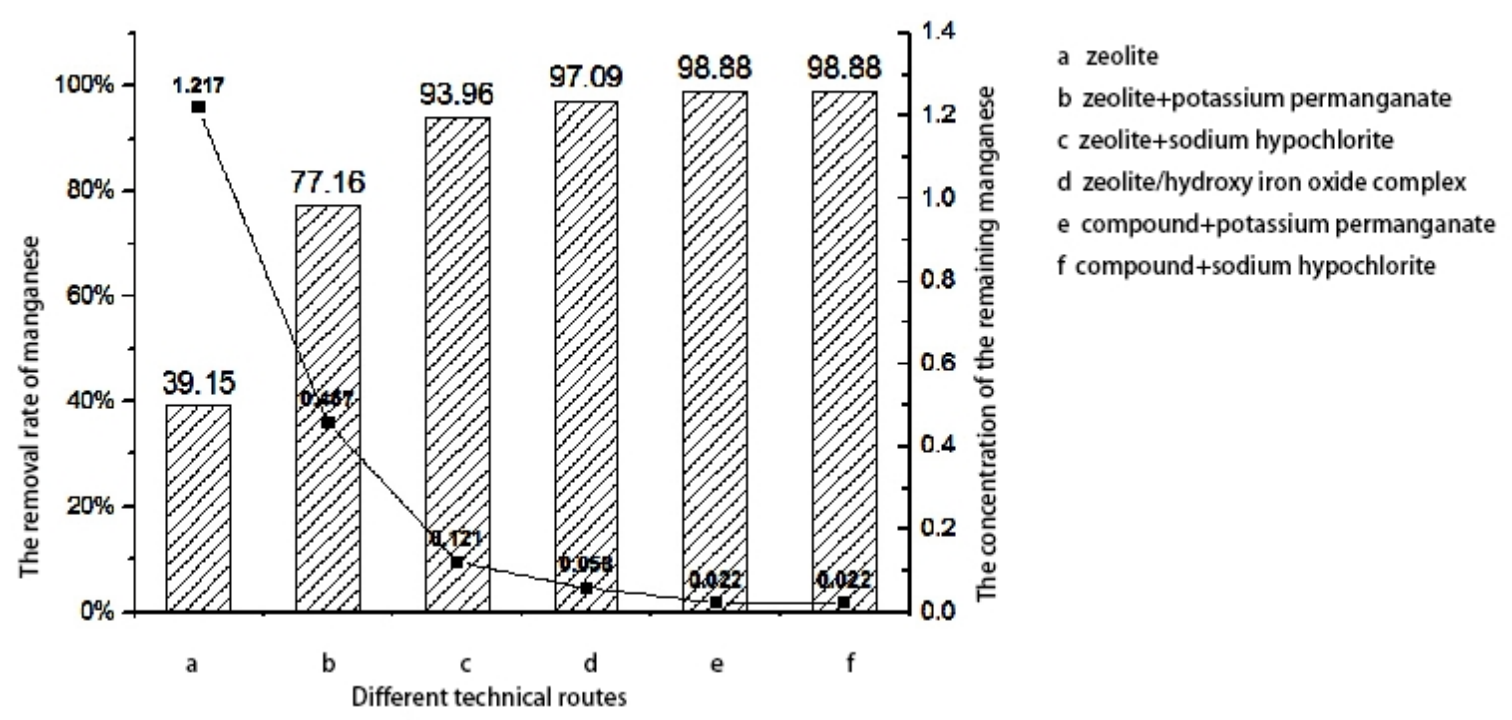

Fig.6 the effect of different technical routes on the removal of manganese ions

Fig.6 shows that the oxidation method can effectively improve the removal rate of manganese, which is better than the adsorption of pure zeolite. However, the effect of removing the manganese by the adsorption of the zeolite / hydroxyl iron oxide compound is better than that of taking the zeolite as the catalyst. It can be seen that the method of using hydroxyl iron oxide loaded on zeolite can 
effectively improve the removal efficiency of manganese. In the presence of oxidants, the removal efficiency is close to $99 \%$, and the reaction speed is faster.

Because the zeolite / hydroxyl iron oxide itself has a significant adsorption effect, when the addition of oxidants, it can effectively reduce the dosage of oxidants. The theoretical removal of $1 \mathrm{mg}$ of iron and manganese requires the addition of $2.874 \mathrm{mg}$ of potassium permanganate and $1.53 \mathrm{mg}$ of sodium hypochlorite. The best dosing ratios in the test were 2.0 and 1.5 , respectively.

At the same time, because the zeolite / hydroxy iron oxide compound has a better settling properties, all the catalyst and suspended matter are settling in the bottom of the beaker after the reaction has been completed. The upper layer of water in the beaker is clear, and when the water sample is injected into the color tube, only a small amount of solids are removed from the filter. Therefore, different from the traditional filtration method, the manganese removal by contact oxidation of manganese sand is usually adopted at the end of the water treatment process. This test indicates that a method of removing manganese by zeolite / hydroxyl iron oxide can be used in the coagulation sedimentation stage. In the coagulation sedimentation tank, the manganese was removed by slow agitation, adding catalyst, zeolite / hydroxyl iron oxide composite, coagulant and oxidant.

\section{Conclusions}

The supported hydroxyl iron oxide catalyst can effectively enhance the manganese removal effect of the carrier. Its treatment effect is much better than the zeolite catalyst alone.

Zeolite / hydroxy iron oxide complex is a very strong adsorbent, the water manganese has a strong adsorption effect. It can meet the drinking water standard in a separate adsorption process. When adding a small amount of oxidant, you can make the concentration of manganese in water to trace, and the reaction speed is extremely fast.

Zeolite / hydroxy iron oxide complex catalyst when the reaction speed is extremely fast, within 30min can be completed.

Because of the strong settling properties of the composites, the suspended matter can be quickly deposited to the bottom of the cup. Therefore, this method can be applied to the coagulation and sedimentation stage in the water treatment process.

\section{References}

[1] Guibai Li, Chao Liu. Removal of iron and manganese from groundwater [M]. Beijing: China Construction Industry Press, 1987 (in Chinese).

[2] Guibai Li. Some new developments of groundwater iron and manganese removal technology. Water supply and drainage, 1983,9 (3): 1-4 (in Chinese).

[3] Vaishya $R$ C.Coated sand filtration: an emerging technology for water treatmeng.AQUA,2003,52(4):299-306.

[4] Shidong Yang, Yuling Li, Dongmei Wang. Study on the adsorption of hydroxyl oxide on the adsorption of phenol [J]. Water treatment technology, 2010,36 (6): 69-72 (in Chinese).

[5] Min Li, Na Li, Qingle Zhang. Adsorption properties of modified zeolite / hydroxy iron oxide composite on methylene blue [J]. Energy and environmental protection, 2013,27 (5): 34-40 (in Chinese).

[6] Huang Yao, Jinping Peng, Qian Yu. Preparation of diatomite load hydroxyl oxide and its phosphorus removal properties [J]. Inorganic salt industry, 2014, 43(5):24-27 (in Chinese).

[7] Xiaotie Zhou, Xuegang Liu, Shiqun Sun. Organic modified bentonite load hydroxyl ferric oxide containing chromium (VI) wastewater treatment [J]. Journal of chaohu college, 2014 (3) : 59-62 (in Chinese). 